\title{
Dual Window Phenomenon for Surface Plasmon Resonance Sensor Based on Plastic Optical Fiber
}

\author{
Yu-Cheng Lin \\ Electronic Engineering Department, Ming Chuan University, \\ 5 De-Ming Rd., Gui-Shan District, Taoyuan 33348, Taiwan \\ (Received November 16, 2019; accepted June 23, 2020)
}

Keywords: surface plasmon resonance, plastic optical fiber, sensor, thin film

Surface plasmon resonance (SPR) sensors are attracting considerable attention for their use in biomaterials inspection, chemical detection, and physical testing. This paper presents an experimental investigation on an SPR-based plastic optical fiber (POF) refractive index sensor. The sensor was fabricated using a POF in which some of the core was removed by polishing and a $40 \mathrm{~nm}$ gold thin film was deposited on the polished surface. The refractive index sensor was used to measure analytes with refractive indices in the range from 1.330 to 1.456 . Two windows, an SPR window and a refraction window, were observed in the optical transmission spectrum, where the SPR window from 650 to $700 \mathrm{~nm}$ and the refraction window from 760 to $780 \mathrm{~nm}$ show a strong linear relationship between the SPR wavelength and the normalized transmitted intensity. Sensitivities of $328 \mathrm{~nm} /$ RIU and $-0.83 /$ RIU (RIU, refractive index unit) were achieved for the SPR and refraction windows, respectively. We also discuss the merits of both windows for biomolecule sensing.

\section{Introduction}

Over the past decade, surface plasmon resonance (SPR)-based sensors have been used in a wide range of physical, ${ }^{(1)}$ chemical, ${ }^{(2,3)}$ and biological sensing schemes. ${ }^{(4,5)}$ The German physicist Kretschmann used a prism substrate covered with a metal thin film of several tens of nanometers thickness to achieve SPR detection. ${ }^{6}{ }^{6}$ Thereafter, SPR technology started to develop rapidly. Nowadays, prism-based SPR sensors have been commercialized and are widely used in surface analysis, as well as in chemical and biological detection. ${ }^{(7,8)}$ Recently, another approach with fiber-based SPR sensors has also been attracting considerable attention for use in biomaterial inspection, ${ }^{(9)}$ physical tests, and so forth. Prism-based SPR sensors are large devices with long turning arms, and their bulky structure makes them less than ideal for the in situ monitoring of industrial and environmental processes. ${ }^{(10,11)}$ Compared with prism-based SPR sensors, optical-fiber-based SPR sensors are fundamentally simpler in construction, less costly, more portable, and more convenient to use. In addition, optical-fiber-based SPR sensors

*Corresponding author: e-mail: yclin@mail.mcu.edu.tw

https://doi.org/10.18494/SAM.2020.2712 
enable the real-time and on-line detection of changes in the refractive index of a bulk medium because wavelength spectrum integration is adopted. There have been many studies on SPR-based optical fiber sensors. ${ }^{(12-15)}$ To enhance the sensitivity to a certain physical parameter, the guiding properties of the optical fiber must be weakened. The side polishing method, which requires the removal of part of the cladding and core, is difficult to implement. In addition, silica optical fibers are fragile and difficult to handle during surface modification processes to realize biosensors.

Plastic optical fibers (POFs) are suitable for niche applications involving short-distance connections and are being actively considered for use in networks in homes and small offices. New research is also opening up a number of other significant possibilities. ${ }^{(16,17)}$ POFs are especially advantageous for low-cost sensing systems owing to their excellent flexibility, easy manipulation, outstanding numerical aperture, and large diameter, and the ability of plastic to withstand smaller bending radii than glass. Compared with silica fibers, POFs have a more flexible, effective, and robust performance in sensing and a higher biocompatibility. ${ }^{(16,18)}$ In this work, a gold thin film was deposited on a side-polished POF, and wavelength monitoring was performed for a wide range of wavelengths. The refractive index sensor was demonstrated to be suitable for dual-window sensing, with one window due to SPR and the other window due to refraction. We also discuss the merits of both windows for biomolecule sensing. To the best of our knowledge, this is the first paper to discuss a dual-window SPR sensor.

\section{Operation Principle}

\subsection{SPR}

During optical fiber transmission, light coupled into the fiber core undergoes total internal reflection at the interface of the core and cladding of the fiber. The fiber core can be used as a support of a metal film deposited on the core for SPR detection. In the optical phenomenon of SPR, a p-polarized electromagnetic wave, namely, a surface plasmon wave (SPW), propagates along the metal-dielectric interface. When p-polarized light is incident in such a way that the propagation constant of the evanescent wave is equal to that of the SPW, light is strongly absorbed as a result of the transfer of energy, and the output signal exhibits a sharp dip at a particular wavelength known as the resonance wavelength. The optical fields associated with the SPR penetrate and decay exponentially into both media and thus can be used to sense the dielectric region near the interface. SPR occurs when the real part of the thin-film permittivity is negative and higher in magnitude than both the imaginary and real parts of the permittivity of the material surrounding the thin film (i.e., the optical waveguide and the surrounding medium in contact with the thin film). The resonance condition is given by ${ }^{(19)}$

$$
\frac{2 \pi}{\lambda} \cdot n_{c} \cdot \sin \theta=\frac{2 \pi}{\lambda} \cdot \sqrt{\frac{\varepsilon_{m r} \cdot n_{s}{ }^{2}}{\varepsilon_{m r}+n_{s}{ }^{2}}} .
$$


The term on the left-hand side is the propagation constant of the evanescent wave generated as a result of attenuated total reflection, where $\theta$ is the angle of the light incident to the core-metal interface and $n_{c}$ is the refractive index of the fiber core. The right-hand term is the SPW propagation constant with $\varepsilon_{m r}$ the real part of the dielectric constant of the metal and $n_{s}$ the refractive index of the sensing dielectric layer. This matching condition of propagation constants is highly sensitive to even a tiny change in dielectric constant, which makes this technique a robust tool for sensing a surrounding dielectric of analyte or for detecting bio-molecules.

\subsection{Refractive index}

Among the many plastics that can be used as an optical fiber, we use poly(methyl methacrylate (PMMA) for the current POF sensor. The refractive index of PMMA varies with wavelength according to the Sellmeier dispersion relation, ${ }^{(20)}$

$$
n_{c}(\lambda)=\sqrt{1+\frac{a_{1} \lambda^{2}}{\lambda^{2}-b_{1}^{2}}+\frac{a_{2} \lambda^{2}}{\lambda^{2}-b_{2}^{2}}+\frac{a_{3} \lambda^{2}}{\lambda^{2}-b_{3}^{2}}}
$$

where $\lambda$ is the wavelength in $\mu \mathrm{m}$ and $a_{1}, a_{2}, a_{3}, b_{1}, b_{2}$, and $b_{3}$ are Sellmeier coefficients with values of $a_{1}=0.99654, a_{2}=0.18964, a_{3}=0.00411, b_{1}=0.00787 \mu \mathrm{m}, b_{2}=0.02191 \mu \mathrm{m}$, and $b_{3}=0.385727 \mu \mathrm{m}$. According to the Drude formula, the dielectric function of any metal can be written as ${ }^{(21)}$

$$
\varepsilon_{m}(\lambda)=1-\frac{\lambda_{c} \lambda^{2}}{\lambda_{p}^{2}\left(\lambda_{c}+i \lambda\right)}
$$

where $\lambda_{p}$ and $\lambda_{c}$ represent the plasma and collision wavelengths, respectively. For example, the values of gold are $\lambda_{p}=1.6826 \times 10^{-7} \mathrm{~m}$ and $\lambda_{c}=8.9342 \times 10^{-6} \mathrm{~m}$. On the basis of the electromagnetic theorem, the dielectric constant of a metal can be expressed in terms of the refractive index as

$$
\varepsilon_{m}=\left(n_{m r}+i k_{m i}\right)^{2}
$$

where $n_{m r}$ and $k_{m i}$ are the real and imaginary parts of the refractive index of the metal, respectively. The refractive index of a metal is also wavelength-dependent.

The refractive indices of PMMA and gold calculated at wavelengths of the visible and infrared bands are shown in Fig. 1. It is observed that upon increasing the wavelength of incident light from 0.4 to $1.8 \mathrm{~mm}$, the refractive index decreases from 1.506 to 1.473 , which is also well known as material dispersion. In the case of light incident on a metal, the reflectivity from the metal surface is extremely high (almost 100\%), especially at grazing incidence $\left(\theta \approx 90^{\circ}\right)$, because of the large extinction coefficient (imaginary part $\left.k_{m i}\right)$ and the small real part 


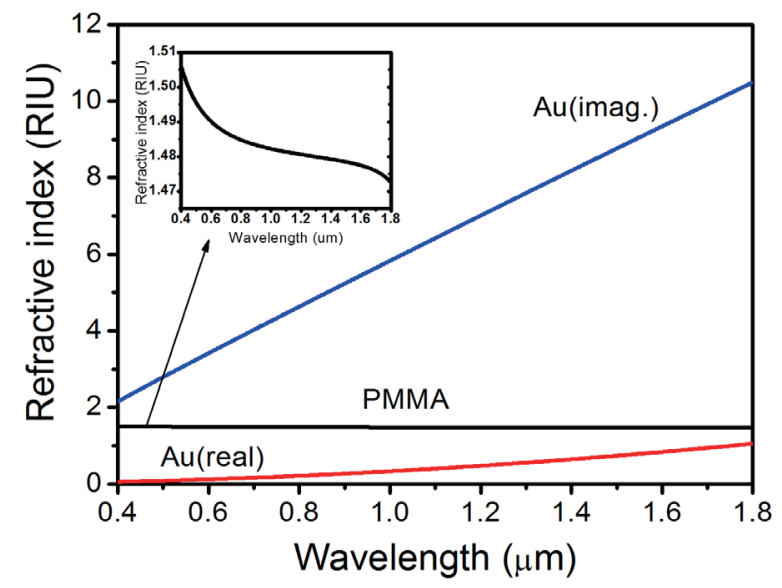

Fig. 1. (Color online) Refractive indices of PMMA and gold calculated at wavelengths of visible and infrared bands.

$n_{m r}$ of the metal. In fact, if the refractive index of a metal is a pure imaginary number, wave propagation in the metal layer is always evanescent. However, if the metal layer is thin enough, the tail energy of the evanescent field will penetrate the sensing area. When the sensed object is changed slightly, it will affect the evanescent field. Under the situation of p-polarized light incident at a specific angle at a certain wavelength, the free electrons of the metal resonate with the wave vector of the incident light to induce SPR. According to Fig. 1, gold (Au) with large imaginary and small real parts of the refractive index has the dielectric properties of a metal. Thus, SPR can be generated with a Au thin-film layer.

\section{Experiment}

\subsection{Fabrication of sensor}

A step-index POF with a diameter of $1 \mathrm{~mm}$, a core diameter of $980 \mu \mathrm{m}$, and a cladding of $20 \mu \mathrm{m}$ was purchased from Grace Plastic Optical Fiber Co. Since the core diameter of the POF is 100 times larger than that of a single-mode fiber (SMF) with a $9.8 \mu \mathrm{m}$ core, the power transmitted in the POF is much higher than that in the SMF. To access the core of the POF, a side polishing technique was adopted. The process involved four steps. First, the POF was roughly polished using a diamond film of $30 \mu \mathrm{m}$ grain size for $35 \mathrm{~s}$. Next, it was polished with two thinner diamond films of 3 and $1 \mu \mathrm{m}$ grain sizes for 80 and $500 \mathrm{~s}$, respectively, to smooth the sensing region. The final polishing was performed with a film with an effective particle size of $0.05 \mu \mathrm{m}$ for $600 \mathrm{~s}$ to obtain a flat finished surface. The side-polished surface of the POF was about $10 \mathrm{~mm}$ in length and $0.5 \mathrm{~mm}$ in depth, which were confirmed by optical microscopy (Olympus, BH). A side view of the polished surface over half of its length $(5 \mathrm{~mm})$ is shown in Fig. 2(a). A progressive bevel is clearly formed on the polished surface.

The two main metals used to induce SPR are silver (Ag) and gold (Au). Silver has a narrow and sharp SPR curve, whereas gold shows a higher shift in resonance than other metals. In 


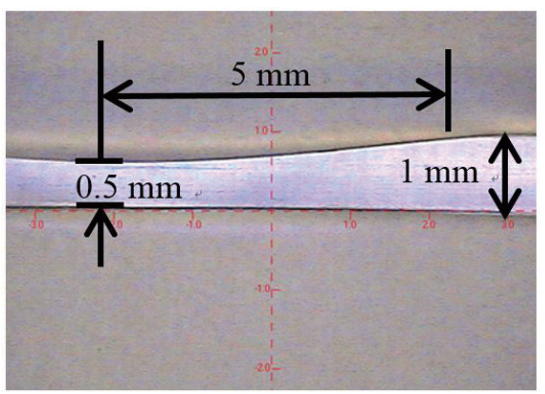

(a)

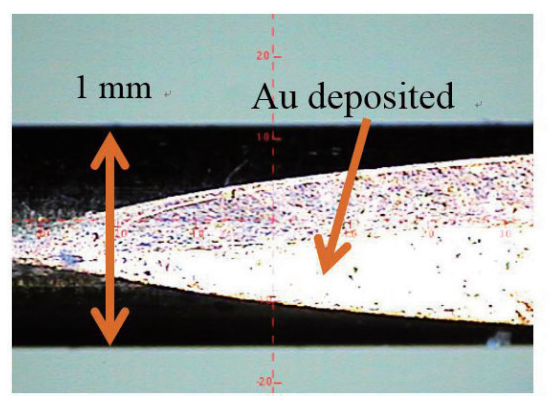

(b)

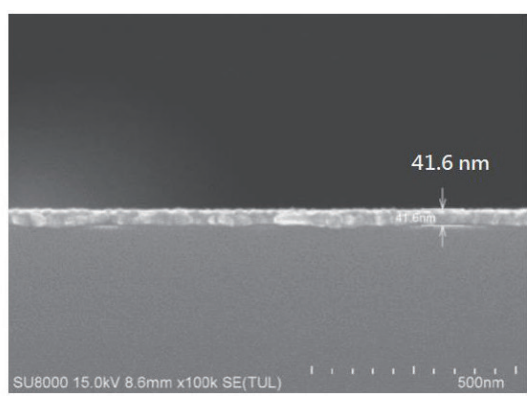

(c)

Fig. 2. (Color online) (a) Side view of POF after polishing, (b) top view of POF after gold deposition, and (c) SEM image of gold thin film on POF.

addition, gold is not easily oxidized, making it suitable for long-term use. In our experiment, the gold thin film was used to induce SPR. The film was deposited on the polished surface using a DC sputtering system (ULVAC). During deposition, the vacuum chamber was evacuated to $4 \times 10^{-2}$ torr and the background pressure was $2 \times 10^{-5}$ torr. The top view of the POF after polishing and deposition has a conical shape as shown in Fig. 2(b). The Au thin film was examined by scanning electron microscopy (SEM) (S-3000H, Hitachi) at $15 \mathrm{kV}$ and its thickness was found to be about $41.6 \mathrm{~nm}$, as shown in Fig. 2(c).

\subsection{Analyte samples}

A series of analytes with different refractive indices were prepared in order to characterize the refractive response of the device. The analytes comprised glycerol (Huaho Chemical Co.) with concentrations from 0 to $90 \%$ in steps of $10 \%$ in pure water. Next, the refractive indices of the analytes were determined using an R-5000 handheld refractometer (ATAGO). We first placed the refractometer in a location with enough light and calibrated the instrument with distilled water. Next, we placed a drop of the analyte between two prisms, and a cross wire was placed at the boundary of the dark and bright regions by the fine adjustment of the radial arm, then readings were taken. The refractive indices for the solutions with different glycerol concentrations were 1.334 (0\%), 1.344 (10\%), $1.358(20 \%), 1.372$ (30\%), $1.386(40 \%), 1.400(50 \%)$, $1.414(60 \%), 1.428(70 \%), 1.442(80 \%)$, and $1.456(90 \%)$.

\subsection{Spectrum measurement}

A typical optical transmission measurement setup was used to collect the spectra of the light through the sensor. A schematic of the experimental setup is shown in Fig. 3. We used halogen light with wavelengths of $400-1800 \mathrm{~nm}$ as the input source (4303B, ANDO) and an optical spectrum analyzer (OSA; AQ-6315, ANDO) as a receiver to measure the SPR spectrum of the sensor. The spectrum measurement procedure was as follows. First, the spectrum of air was recorded as a reference to normalize the SPR sensor spectra for the analytes. The reference air spectrum provided an effective initial spectrum, attributed to the halogen light spectra, the 


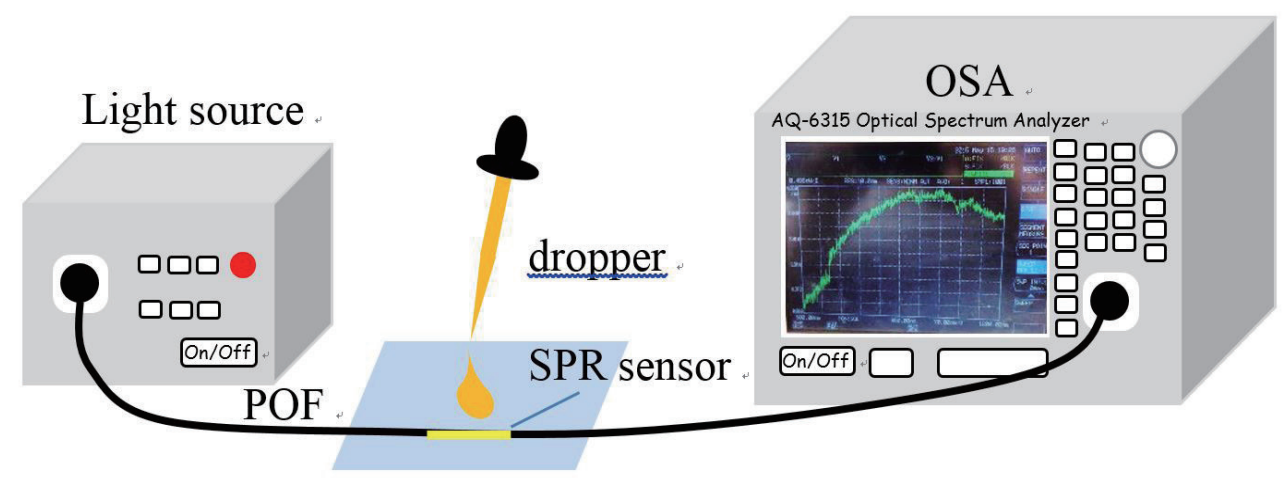

Fig. 3. (Color online) Setup for optical transmission spectrum measurement.

POF absorbance spectrum, and the OSA spectral sensitivity. Then, we introduced drops of an analyte into the sensing region of the SPR sensor without disturbing the POF and recorded the spectra of the transmitted light. Finally, these spectra were normalized with the reference air spectrum.

\section{Results and Discussion}

\subsection{POF absorption spectrum}

The absorption of PMMA is mainly caused by the asymmetrical bending vibration of a methyl group $\left(\mathrm{CH}_{3}\right)$ and a carbonyl group, which leads to the absorption band and must be avoided when utilizing a POF. ${ }^{(2)}$ In Fig. 4, the transmission spectrum of the POF was measured and compared with that of an M74L01 glass optical fiber (GOF; Thorlabs) with a core diameter of $400 \mu \mathrm{m}$, which is only about half that of the POF, so the overall transmission light intensity of the POF is much higher than that of the GOF. The wavelength in which the optical intensity of the POF is greater than $100 \mathrm{nW}$ ranges from 500 to $850 \mathrm{~nm}$, while that of the GOF ranges from 600 to $1100 \mathrm{~nm}$ with no absorption on the band. In addition, note that the POF has a strong absorption band from 880 to $920 \mathrm{~nm}$ and above $960 \mathrm{~nm}$, whereas the light of these wavelengths is almost completely absorbed by the POF. The absorption band must be avoided when the sensor is used.

\subsection{SPR and refraction windows}

Figure 5 shows the SPR spectra obtained from the refractive index sensor for analytes with refractive indices varying from 1.344 to 1.456 . There are two transmission windows in the spectra. One is the SPR window, which is from 650 to $700 \mathrm{~nm}$ and highlighted in yellow, where the dip at a particular wavelength in each curve is equal to the resonance wavelength $\left(\lambda_{\text {res }}\right)$. The larger the refractive index, the longer the resonance wavelength will be. The red 


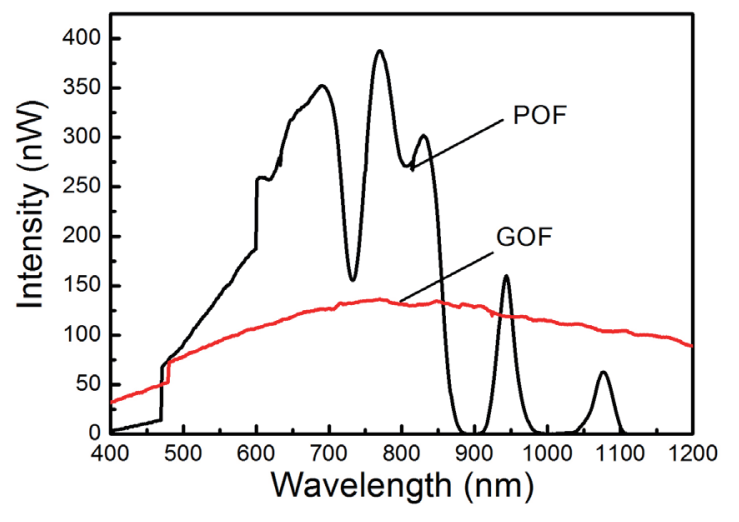

Fig. 4. (Color online) Transmission spectra for POF and GOF.

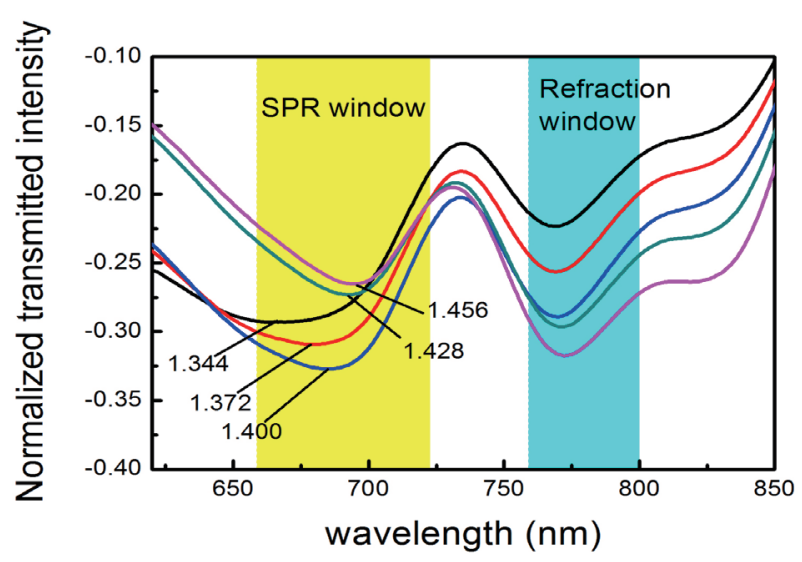

Fig. 5. (Color online) SPR spectra for analytes with refractive indices from 1.344 to 1.456 .

shift in the SPR wavelength with increasing refractive index of analytes is similar to the results presented in Ref. 21. The other window is the refraction window, which is from 760 to $780 \mathrm{~nm}$ and highlighted in blue, where the normalized transmitted intensity decreases with increasing refractive index of the analyte at a fixed wavelength of $770 \mathrm{~nm}$. In fact, there is also good sensing performance in the wavelength range of 770 to $800 \mathrm{~nm}$.

Figure 6 presents the variation in SPR wavelength for the analytes with different refractive indices from 1.334 to 1.456 . In the SPR window, the sensor shows good linearity between the refractive index and the SPR wavelength when the refractive index of the analyte is less than 1.428. The linear fitting results gave a slope of 328.0 and a coefficient of determination, $R^{2}$, of 0.94 . The wavelength shifts by $31.5 \mathrm{~nm}$ when the refractive index of the analyte increases by 0.098. From the slope of the line, the sensitivity of the sensor is $328 \mathrm{~nm} / \mathrm{RIU}$. For the refraction window, the linear fitting results gave a slope of 30.3 with $R^{2}$ of 0.93 , although there is only a tiny shift in the wavelength of the dip of about $4.5 \mathrm{~nm}$ for the same increase in refractive index. It was considered that no SPR occurred in the refraction window.

The variation in normalized transmitted intensity for the two windows for the analytes with different refractive indices from 1.334 to 1.456 is shown in Fig. 7. The refraction window clearly shows a linear relationship between the transmitted intensity and the refractive index, where $R^{2}$ is 0.94 . This phenomenon is due to the Fresnel reflection when an optical wave passes from one medium to another with a different refractive index in the sensing region. The transmitted power is affected by the refractive index of the analyte. The lower the difference in refractive index between the two media at the interface, the higher the refractive power and the lower the transmitted power will be. In the SPR window, a linear fit with $R^{2}$ of 0.95 is achieved for refractive indices of the analyte of less than 1.428 and the normalized transmitted intensity increases largely from -0.34 to -0.27 for higher refractive indices above 1.430 . This can be explained using the SPR condition given by Eq. (1). The term on the left-hand side is the propagation constant of the evanescent wave generated as a result of the attenuated total reflection of the light incident at angle $\theta$. The right-hand term is the SPW propagation constant 


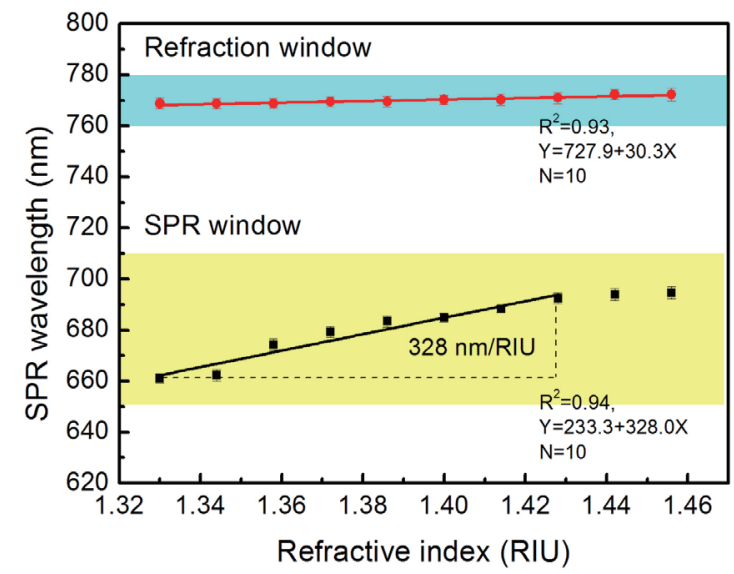

Fig. 6. (Color online) Variation in SPR wavelength for analytes with refractive indices from 1.334 to 1.456 .

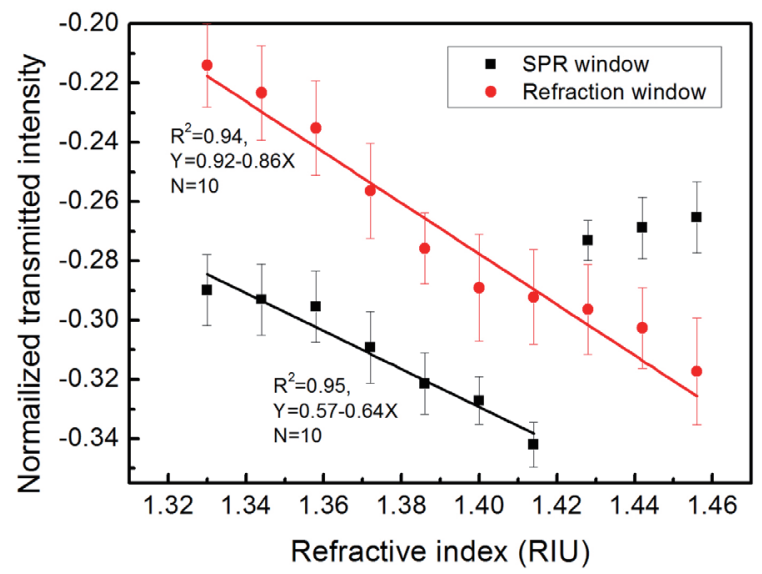

Fig. 7. (Color online) Variation in normalized transmitted intensity for analytes with refractive indices from 1.334 to 1.456 .

with $\varepsilon_{m r}$ the real part of the dielectric constant of the metal $\left(\varepsilon_{m}\right)$ and $n_{s}$ the refractive index of the analyte. This matching condition of the propagation constants is determined by the properties of the materials of the devices such as the refractive index of PMMA $\left(n_{c}\right)$, the dielectric constant of the metal film $\left(\varepsilon_{m r}\right)$, and the incident angle $(\theta) .{ }^{(22,23)}$ In general, the propagation constant of an evanescent wave is slightly larger than that of an SPW. By adjusting the incident angle, the left-hand term can be slightly decreased to match the right-hand term. However, the right-hand term will be greater than the left-hand term if an analyte with a large refractive index is added. As a result, SPR cannot occur at any incident angle. Therefore, to obtain the limit of $n_{s}$, the above equation is rewritten as

$$
n_{c} \cdot \sin \theta \geq \sqrt{\frac{\varepsilon_{m r} \cdot n_{s}{ }^{2}}{\varepsilon_{m r}+n_{s}{ }^{2}}} .
$$

By rearranging Eq. (5), the maximum limit of $n_{s}$ can be written as

$$
n_{s} \leq \sqrt{\frac{\varepsilon_{m r} \cdot n_{c}{ }^{2} \cdot \sin ^{2} \theta}{\varepsilon_{m r}-n_{c}{ }^{2} \cdot \sin ^{2} \theta}} .
$$

The maximum refractive index of the analyte $\left(n_{s}\right)$ at which the SPR is excited for all operating wavelengths obtained from Eq. (6) is shown in Fig. 8. According to the calculation result, if an analyte with a higher $n_{s}$ is added dropwise to the sensor, SPR will not occur. The maximum value of $n_{s}$ is about 1.40 at $700 \mathrm{~nm}$, and this is why the normalized transmitted intensity increases and the SPR becomes less evident when the analytes with refractive indices of 1.428 and 1.456 are applied to the SPR sensor as shown in Figs. 5 and 7. The SPR sensor is a 


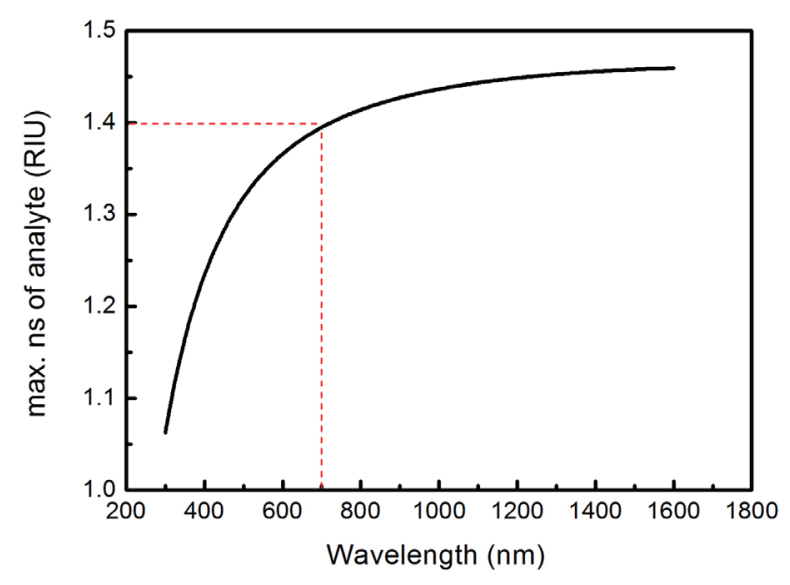

Fig. 8. (Color online) Maximum refractive index of analyte $\left(n_{s}\right)$ at which SPR can be obtained from Eq. (6).

wavelength investigation technology depending on the refractive index of the analyte. However, there is also a limitation of SPR for an analyte with a high refractive index, as shown in Eq. (6), where SPR does not occur.

In general, trace amounts of biomolecules are usually stored in a buffer solution for biomolecule detection. SPR sensing is affected by two mechanisms. One is the wavelength shift induced by the refractive index of the buffer solution, and the other is the interaction of the biomolecule with the surface of the SPR sensor, such as by adsorption or probe collection. In the current SPR sensing method, cross-linking phenomena such as the capture of the biomolecule and the variation in the refractive index of the buffer solution are examined only on the basis of the resonance wavelength shift of the SPR sensor. However, the refractive index of the buffer solution is sensitive to temperature, which will make SPR unable to accurately distinguish real biomolecule capture signals. The refraction window in this study can be used to sense the effects of temperature changes. In the future, the sensing of SPR can be used to eliminate the effect of the buffer solution and obtain the actual interaction between biomolecules and the SPR surface.

\section{Conclusion}

We have experimentally demonstrated a refractive index sensor based on SPR with a side-polished POF coated with a gold thin-film layer. The refractive index sensor was used to measure analytes with refractive indices in the range from 1.334 to 1.456 . Two windows, an SPR window and a refraction window, were observed in the optical spectrum. The SPR window shows a strong linear relationship between the SPR wavelength and the refractive index of the analyte, and the refraction window shows a normalized transmitted intensity linear to the refractive index of the analyte. Sensitivities of $328 \mathrm{~nm} / \mathrm{RIU}$ for the SPR window and $-0.83 / \mathrm{RIU}$ for the refraction window were observed. We also discussed the merits of both windows for 
biomolecule sensing. Our proposed sensor has the merits of robustness, low cost, and suitability for in vivo testing, and has the ability to be applied to chemical and biological sensing.

\section{Acknowledgments}

The author would like to thank the Ministry of Science and Technology for financial support under contract No. MOST 108-2221-E-130-014. The author would also like to thank the Graduate Institute of Electro-optical Engineering, Tatung University, for supplying the sputtering equipment and Mr. Jiacheng Wu and Mr. Hongyi Li for assistance in the experiment.

\section{References}

1 Y. Nishijima, Y. Hashimoto, A. Balčytis, G. Seniutinas, and S. Juodkazis: Sens. Mater. 29 (2017) 1233. https:// doi.org/10.18494/SAM.2017.1582

2 R. Ahmed: Int. J. Photoenergy 2009 (2009). https://doi.org/10.1155/2009/150389

3 W. Daniyal, S. Saleviter, and Y. W. Fen: Sens. Mater. 30 (2018) 2023. https://doi.org/10.18494/SAM.2018.1952

4 N. Soh, T. Watanabe, Y. Asano, and T. Imato: Sens. Mater. 15 (2003) 423. https://doi.org/10.1002/ elan.200302809

5 P. Singh, T. Onodera, Y. Mizuta, K. Matsumoto, N. Miura, and K. Toko: Sens. Mater. 19 (2007) 261.

6 H. R. E. Kretchmann: Z. Naturforsch 23 (1968) 2135. https://doi.org/10.1515/zna-1968-1247

7 P. K. Maharana and R. Jha: Sens. Actuators, B 169 (2012) 161. https://doi.org/10.1016/j.snb.2012.04.051

8 T. Tang: Optik Int. J. Light Electron. Opt. 124 (2013) 4526. https://doi.org/10.1016/j.ijleo.2013.01.052

9 Y. Jin, K. H. Wong, and A. M. Granville: Colloids Surf. Physicochem. Eng. Aspects 492 (2016) 100. https:// doi.org/10.1016/j.colsurfa.2015.11.025

10 B. Lee, J.-H. Park, J.-Y. Byun, J. H. Kim, and M.-G. Kim: Biosens. Bioelectron. 102 (2018) 504. https://doi. org/10.1016/j.bios.2017.11.062

11 Y. Yuan, L. Ding, and Z. Guo: Sens. Actuators, B 157 (2011) 240. https://doi.org/10.1016/j.snb.2011.03.056

12 Y.-C. Lin, Y.-c. Tsao, W.-H. Tsai, T.-S. Hung, K.-S. Chen, and S.-C. Liao: Sens. Actuators, B 133 (2008) 370. https://doi.org/10.1016/j.snb.2008.02.051

13 T. Woo-Hu, L. Yu-Cheng, T. Jiu-Kai, and T. Yu-Chia: Opt. Laser Technol. 42 (2010) 453. https://doi. org/10.1016/j.optlastec.2009.09.008

14 Y.-C. Tsao, Y.-C. Lin, W.-H. Tsai, and M.-S. Wu: Sens. Actuators, A 166 (2011) 9. https://doi.org/10.1016/ j.sna.2010.12.015

15 S. Shukla, N. K. Sharma, and V. Sajal: Sens. Actuators, B 206 (2015) 463. https://doi.org/10.1016/j.snb.2014.09.083

16 N. Cennamo, F. Chiavaioli, C. Trono, S. Tombelli, A. Giannetti, F. Baldini, and L. Zeni: Sensors 16 (2016) 196. https://doi.org/10.3390/s16020196

17 Y. Hu, Y. Hou, A. Ghaffar, and W. Liu: IEEE Access (2020). https://doi.org/10.1109/ACCESS.2020.2993707

18 N. Cennamo, D. Massarotti, L. Conte, and L. Zeni: Sensors 11 (2011) 11752. https://doi.org/10.3390/s111211752

19 B. D. Gupta and R. Kant: Opt. Laser Technol. 101 (2018) 144. https://doi.org/10.1016/j.optlastec.2017.11.015

20 S. N. Kasarova, N. G. Sultanova, C. D. Ivanov, and I. D. Nikolov: Opt. Mater. 29 (2007) 1481. https://doi. org/10.1016/j.optmat.2006.07.010

21 Y. S. Dwivedi, A. K. Sharma, and B. D. Gupta: Appl. Opt. 46 (2007) 4563. https://doi.org/10.1364/ AO.46.004563

22 N. A. S. Omar, Y. W. Fen, S. Saleviter, Y. M. Kamil, W. M. E. M. M. Daniyal, J. Abdullah, and M. A. Mahdi: Sens. Actuators, A 303 (2020) 111830. https://doi.org/10.1016/j.sna.2020.111830

23 Y. Zhao, S. Gan, G. Zhang, and X. Dai: Results Phys. 14 (2019) 102477. https://doi.org/10.1016/j.rinp.2019.102477 


\section{About the Author}

Yu-Cheng Lin received his B.S. degree from Tatung University, Taiwan, in 1989 and his M.S. and Ph.D. degrees from the Graduate Institute of Electrical Engineering, Tatung University, in 1991 and 1995, respectively. From 1997 to 2000, he was an assistant professor at Vanung University, Taiwan. From 2000 to 2001, he was a senior engineer at Foxconn Ltd. Co., Taiwan. Then, he worked as an R\&D manager for optical fiber passive products at Wanshih Ltd. Co., Taiwan, from 2001 to 2004. Since 2004, he has been an associate professor at Ming Chuan University. His research interests are in optical fiber devices and sensors. (yclin@mail.mcu.edu.tw) 